\title{
The Rate of Convergence of Hermite Function Series
}

\author{
By John P. Boyd
}

Abstract. Let $\alpha>0$ be the least upper bound of $\gamma$ for which

$$
f(z) \sim O\left(e^{-q|z| \gamma}\right)
$$

for some positive constant $q$ as $|z| \rightarrow \infty$ on the real axis. It is then proved that at least an infinite subsequence of the coefficients $\left\{a_{n}\right\}$ in

$$
f(z)=e^{-z^{2} / 2} \sum_{n=0}^{\infty} a_{n} H_{n}(z),
$$

where the $H_{n}$ are the normalized Hermite polynomials, must satisfy certain lower bounds. The theorems show two striking facts. First, the convergence rate of a Hermite series depends not only upon the order $\rho$ for an entire function or the location of the nearest singularity for a singular function as for a power series but also upon $\alpha$, thus making the convergence theory of Hermitian series more complicated (and interesting) than that for any ordinary Taylor expansion. Second, the poorer the match between the asymptotic behavior of $f(z)$ and $\exp \left(-1 / 2 z^{2}\right)$ the poorer the convergence of the Hermite series will be.

1. Introduction. Series representations of the form

$$
f(z)=\sum_{n=0}^{\infty} a_{n} h_{n}(z),
$$

where

$$
h_{n}(z)=e^{-z^{2} / 2} H_{n}(z)
$$

is the $n$th normalized Hermite function and $H_{n}(z)$ is the $n$th normalized Hermite polynomial, have been used and studied for more than a century, yet important gaps remain in our understanding of such functions. The motive for the present work, the goal of which is to fill some of these gaps, is that Hermite functions are the asymptotic solutions to many interesting physical problems as illustrated by the examples of Boyd [2]. In consequence, an assumed expansion of the form (1.1) is, via Galerkin's method, a powerful way of numerically solving such problems in the near-asymptotic parameter range. To do so efficiently, however, one needs to understand the relationship between the properties of $f(z)$ and its Hermite expansion coefficients as thoroughly as possible.

Some theorems of early workers provide a background for this present article. It is known via a simple integration-by-parts argument given in [2] that as long as $f(z)$

Received March 9, 1979; revised March 21, 1980.

AMS (MOS) subject classifications (1970). Primary 42A56; Secondary 33A65, 65N30.

Key words and phrases. Hermite function series. 
decreases exponentially fast as $|z| \rightarrow \infty$ on the real axis, then the normalized expansion coefficients $\left\{a_{n}\right\}$ must decrease faster than any finite power of $n$-the property of "infinite order convergence". This is consoling because numerical analysts often successfully use algorithms which are of only finite order, i.e., finite difference and finite element methods, but it is not the whole story. If

$$
a_{n} \sim O\left(e^{-p n^{\beta}}\right)
$$

for some positive constants $p$ and $\beta$, then the series (1.1) technically has the property of "infinite order convergence" for all $\beta>0$, but, from a practical standpoint, it matters tremendously whether $\beta=1.0$ or 0.001 .

When $f(z)$ has one or more singularities with $\tau$ being the absolute value of the imaginary part of the location of that singularity which is closest to the real axis, and

$$
f(z) \sim O\left(e^{-q|z| \gamma}\right)
$$

for some positive constant $q$ and constant $\gamma>1$ as $|z| \rightarrow \infty$ within the strip $-\tau<$ $y<\tau$ where $y$ is the imaginary part of $z$, then it is known [4] that (1.3) holds with $\beta=1 / 2$ and $p=2^{1 / 2} \tau$. The purpose of the present article is to provide an upper bound on $\beta$ for entire functions and for singular functions for which (1.4) holds with $\gamma<1$.

In analyzing the various possible cases, a couple of definitions are helpful. The "exponent of decay" $\alpha$ is defined as the least upper bound of $\gamma$ in (1.4) for which (1.4) holds. The "order" $\rho$ is defined, as is conventional in entire function theory, as the least upper bound of $\gamma$ for which

$$
f(z) \sim O\left(e^{q|z| \gamma}\right)
$$

as $|z| \longrightarrow \infty$ in any direction. Seemingly, these two quantities are unrelated since the exponent of convergence $\alpha$ is concerned with how rapidly $f(z)$ becomes small whereas the order $\rho$ is concerned with how rapidly $f(z)$ becomes large, but for a simple function like $\exp \left(-z^{4}\right), \alpha=\rho$, and in most practical applications, this is the case: the exponent of convergence and the order are equal. It is possible, however, for $\rho>\alpha$, and an elementary example will be given in Section 4; a theorem presented in [7] shows that the order cannot be less than the exponent of convergence.

The plan of the work is as follows. Section 2 deals with "super-Gaussian" functions, i.e., those for which $\alpha>2$, while Section 3 proves a similar theorem for $0<$ $\alpha<2$. When $\rho>\alpha$, this can have a profound impact on the convergence of the Hermite series, and this is taken up in Section 4. Results and plans for future work are outlined in the final section.

2. "Super-Gaussian" Functions. The main result of this section is the following:

THEOREM 1. If $f(x)$ is an entire function of order $\rho$ and if the exponent of real axis, decay, defined above, satisfies $\alpha>2$, then at least an infinite subset of the Hermite expansion coefficients $\left\{a_{n}\right\}$ of the function must satisfy

$$
\left|a_{n}\right|>c e^{-d n \sigma+\epsilon}
$$


where $c$ and $d$ are positive constants and $\epsilon>0$ may be arbitrarily small and where

$$
\sigma=\frac{\rho}{2(\rho-1)}
$$

The theorem does not exclude the possibility that individual coefficients may be zero (indeed, if $f(z)$ is symmetric about $z=0$, all the odd-numbered coefficients will be automatically zero) but it does imply that the series as a whole cannot converge too rapidly. One way of demonstrating the theorem is analogous to the proof, given in Titchmarsh [7], of a similar theorem that relates the order of a function to its power series coefficients; in either case, one can show that if the series coefficients decrease more rapidly than allowed by the theorem, $|f(z)|$ could not grow rapidly enough as $|z| \rightarrow \infty$ to be consistent with the assumed order $\rho$. In contrast to the power series case, however, one cannot show that a lower bound in (2.2) is also (changing the sign of $\epsilon$ ) an upper bound because the actual rate of convergence must depend on $\alpha$ as well. If $\alpha$ is negative, for example, the Hermite series will diverge everywhere. The restriction that $\alpha>2$ is necessary because the results of the next section give more precise results for smaller $\alpha$.

Details of the Titchmarsh-like proof will be supplied upon request, but a shorter proof can be based upon Theorem 5.1 of Hille [5], which deals with functions of the operator

$$
\delta_{z} \equiv \frac{d^{2}}{d z^{2}}-z^{2}
$$

whose eigenfunctions are the Hermite functions, i.e.,

$$
\delta_{z} h_{n}(z)=(2 n+1) h_{n}(z) .
$$

Hille showed that $G\left(\delta_{z}\right) f(z)$ is an entire function if and only if $\kappa$, the order of the entire function $G(z)$, is less than $\sigma$ where $\sigma$ is related to $\rho$, the order of $f(z)$ by (2.2). To use Hille's result, let us proceed with a proof by contradiction.

Suppose that

$$
\left|a_{n}\right| \sim O\left(e^{-d n \gamma}\right),
$$

where $\gamma$ is greater than $\sigma$ as given by (2.3), i.e.,

$$
\gamma>\frac{\rho}{2(\rho-1)}+\epsilon
$$

where $\epsilon>0$. If we now choose $G(z)$ to be an entire function of order

$$
\kappa=\gamma-\epsilon / 2
$$

and apply it to the Hermite series term-by-term, using (2.5), it is clear that the Hermite coefficients of $G\left(\delta_{z}\right) f(z)$, call them $\left\{b_{n}\right\}$, satisfy

$$
b_{n} \sim O\left(e^{-d n^{\gamma}+q n}{ }^{\gamma-\epsilon / 2}\right)
$$

for some positive constant $q$ as $n \rightarrow \infty$. Thus,

$$
b_{n} \sim o\left(e^{-\tau(2 n+1)^{1 / 2}}\right)
$$


for any $\tau>0$, therefore establishing from Theorem 2.2 of Hille [4] that the Hermite series of $G\left(\delta_{z}\right) f(z)$ converges everywhere in the finite complex plane, and consequently is an entire function. Since $\kappa>\sigma$, however, this is in contradiction of Hille's Theorem 5.1 [5]. Noting that $\epsilon$ can be taken to be arbitrarily small in (2.6) through (2.8) then proves our Theorem 1.

3. "Sub-Gaussian" Functions. The main result of this section is the following:

THEOREM 2. Let $\alpha$, the exponent of real axis convergence, be the least upper bound of $\gamma$ for which

$$
f(z) \sim O\left(e^{-p|z|^{\gamma}}\right)
$$

for some constant $p$ as $|z| \rightarrow \infty$ on the real axis. Then if $\alpha<2$, at least an infinite subsequence of the normalized Hermite expansion coefficients $\left\{a_{n}\right\}$ of $f(z)$ must satisfy

$$
\left|a_{n}\right|>c e^{-d n^{\sigma+\epsilon}}
$$

for some positive constants $c$ and $d$ where $\epsilon>0$ may be arbitrarily small and where

$$
\sigma=\alpha / 2 \text {. }
$$

The assumptions of this theorem are as striking for what is left unsaid as for what is demanded for the theorem's validity. In particular, Theorem 2 holds even if $f(z)$ has singularities a finite distance off the real axis. For such singular functions, Hille's result, quoted in Section 1, is stronger than the present theorem if $\alpha>1$, but when $\alpha<1$, Hille merely shows that the domain of convergence of the Hermite series is just the real axis itself. Thus, Theorem 2 provides new information for entire functions with $\alpha<2$ and singular functions with $\alpha<1$.

The basic idea of the proof of this theorem is similar to that of the previous theorem except that we will be concerned with the behavior of $f(z)$ and of the Hermite functions only on the real axis. The global strategy is a proof by contradiction. Let us assume

$$
a_{n} \sim O\left(e^{-d n \gamma}\right)
$$

for some positive $d$ with $\gamma>\sigma$ where $\sigma$ is given by (3.3). It will then be shown that $f(z)$ can be bounded by a function which is $O\left(e^{-b|z|^{2 \gamma}}\right)$, which is in contradiction of the assumption that $\alpha(<2 \gamma)$ is the exponent of real axis convergence.

To establish this bound on $f(z)$, we will consider

$$
g(z)=p \sum_{n=0}^{\infty} e^{-q n \gamma}\left|h_{n}(z)\right|,
$$

which is clearly greater than $|f(z)|$ if the positive constant $p$ is sufficiently large. To estimate this function for a given value of $z$, it is convenient to split the sum (3.5) into two parts using a dividing line which is itself a function of $|z|$, i.e., let $N$ be defined by

$$
2 N+1=|z|^{2} / 2,
$$


and let

$$
\begin{gathered}
S_{1} \equiv \sum_{n=0}^{N-1} e^{-q n \gamma}\left|h_{n}(z)\right|, \\
S_{2} \equiv \sum_{n=N}^{\infty} e^{-q n^{\gamma}} .
\end{gathered}
$$

Using the well-known fact that

$$
\left|h_{n}(z)\right| \leqslant 1
$$

for all $n$ and all real $z$, we see

$$
|f(z)|<p\left(S_{1}+S_{2}\right)
$$

for all real $z$. The reason that (3.9) has been used only for $S_{2}$ is that, because of the way $N$ is defined, $z$ is outside the turning points of all the low-order Hermite functions included in $S_{1}$. For $|z|<z_{t}$, the absolute value of the turning points, $h_{n}(z)$ is oscillatory, and thus for some of the Hermite functions included in $S_{2},(3.9)$ is reasonably tight. When $|z|>z_{t}$, as is true for all the $\left\{h_{n}(z)\right\}$ included in $S_{1}$, the Hermite functions are decaying like Gaussians in $|z|$, and we shall ultimately exploit this to show that $S_{1}$ is asymptotically negligible in comparison to $S_{2}$ as $|z|$ and $N$ simultaneously increase.

To proceed further with $S_{2}$, we need the following:

LEMMA 3.1 .

$$
\sum_{n=N}^{\infty} e^{-q n} \sim O\left(N e^{-q(N-1)^{\gamma}}\right)
$$

for any positive constant $q$ and any $\gamma>0$ as $N \rightarrow \infty$.

Proof. The sum

$$
Q_{2} \equiv \sum_{n=N}^{\infty} e^{-q n} \gamma
$$

is a rectangle rule approximation (with unit intervals) to the integral

$$
I_{1}=\int_{N}^{\infty} e^{-q(x-1)^{\gamma}} d x
$$

and since we are approximating $I_{1}$ by rectangles that always lie below the true integrand of $I_{1}$ on each interval, clearly

$$
Q_{2} \leqslant I_{1}
$$

By using integration-by-parts, one can easily derive, just as for the special case of $\gamma=2$ (which is the complementary error function), the asymptotic series for $I_{1}$ as $N \rightarrow \infty$. The leading behavior is

$$
I_{1} \sim \frac{e^{-q(N-1)^{\gamma}}(N-1)^{1-\gamma}}{q \gamma}\left\{1+O\left(\frac{\gamma-1}{q \gamma(N-1)^{\gamma}}\right)\right\},
$$

from which the lemma follows. Replacing $N$ in the lemma by its definition in terms 
of $|z|$, (3.6), then gives at once

$$
S_{2} \sim O\left(|z|^{1 / 2} e^{-p|z|^{2 \gamma}}\right),
$$

where $p\left(=q(1 / 4)^{\gamma}\right)$ is a constant. This estimate for $S_{2}$ then leads to an immediate contradiction with the assumption that $\alpha$, the exponent of real axis decay, is $<2 \gamma$ provided that we can show that $S_{1} \leqslant S_{2}$, at least asymptotically.

The bound on the other sum is the following:

LEMMA 3.2 .

$$
S_{1} \sim O\left(e^{-s|z|^{2}}\right)
$$

Proof. Askey and Wainger [8] show that

$$
\left|h_{n}(z)\right| \sim O\left(e^{-s^{\prime \prime}|z|^{2}}\right)
$$

for some positive constant $s^{\prime \prime}$ if

$$
|z| \geqslant(4 n+2)^{1 / 2}
$$

as is guaranteed by (3.6) for all $h_{n}(z)$ included in the sum $S_{1}$. We can then bound $S_{1}$ itself by multiplying (3.18) by $N$, the total number of Hermite functions in the sum, which gives (3.17) with $s<s^{\prime \prime}$.

From the lemma and (3.16) the theorem itself follows immediately.

Note that all the analysis of this proof involves only the behavior of $f(z)$ and of the Hermite functions themselves on the real axis. Thus, we need not worry about complications such as Stokes lines for the asymptotic expansions we have used and the theorem remains true, without modification, even if $f(z)$ is singular provided the singularities are not on the real axis itself.

4. The Effect of the Order Exceeding the Exponent of Convergence. When the order $\rho$ is greater than the exponent of decay $\alpha$, this can profoundly decelerate the rate of convergence of Hermite series. Since this situation is uncommon in practical problems and since work now in progress may eventually provide a sharp estimate instead of a mere inequality, we will content ourselves with proving the following modest theorem to indicate the possibilities.

THEOREM 3. Suppose the entire function $f(z)$ has the asymptotic form

$$
f(z) \sim e^{-p z^{\alpha}+i q z^{\rho}}
$$

as $|z| \rightarrow \infty$ in the strip $-\tau<y<\tau, x>0$, where $z=x+$ iy for some positive constants $p, q$, and $\tau$. If

$$
\rho>\alpha+1
$$

then the Hermite series of $f(z)$ will converge only on the real axis, and at least an infinite subsequence of the expansion coefficients must decrease more slowly than $O\left(e^{-p^{\prime}(2 n+1)^{1 / 2}}\right)$ for any $p^{\prime}>0$.

Proof. Hille's Theorem 1 [4] shows that a Hermite series can converge within a strip of the complex plane only if it is exponentially decaying as $|z| \rightarrow \infty$ for all $y$ 
within the strip. If (4.1) and (4.2) are satisfied, however, it is trivial to show that $f(z)$ is exponentially increasing as $|x| \rightarrow \infty$ on every line parallel to the real axis in the lower half-plane except for the real axis itself. Thus, the Hermite series for $f(z)$ will have "infinite order" convergence on the real axis and diverge everywhere off the real axis. It is shown by Hille [6] that if the Hermite coefficients are $O\left[\exp \left(-p^{\prime}(2 n+1)^{1 / 2}\right]\right.$ as $n \rightarrow \infty$ for some $p^{\prime}>0$, then the Hermite series will converge in a strip of finite width. Thus,

$$
a_{n} \sim O\left(e^{-p^{\prime}(2 n+1)^{1 / 2}}\right)
$$

for some $p^{\prime}$ is impossible when (4.1) and (4.2) hold.

Hille [4] has already shown that if $\alpha<1$, then the Hermite series of $f(z)$ will converge only on the real axis even if $f(z)$ is entire. The above theorem proves that $\alpha \geqslant 1$ is not a sufficient condition for convergence in a strip of finite width (although it is necessary); it is possible for the Hermite series of an entire function to converge only on the real axis no matter how large $\alpha$, i.e., no matter how rapidly the function decays on the real axis itself.

The function

$$
f(z)=\cos \left(z^{6}\right) e^{-z^{4}}
$$

furnishes a simple example. Though it decays very fast for real $z$, its Hermite coefficients must decrease sufficiently slowly so that its Hermite series diverges everywhere off the real axis. When $\rho<\alpha+1$, however, the Hermite series may converge much more rapidly. For example, consider

$$
g(z)=\cos \left(z^{4.5}\right) e^{-z^{4}}
$$

Hille's theorem can be used to show that the Hermite series of $g(z)$ must converge everywhere in the finite complex plane.

5. Summary and Discussion. In the usual case when the exponent of real axis decay $\alpha$ and the order $\rho$ are equal, Theorems 1 and 2 deal with mutually exclusive cases. The former applies to entire functions that decay faster than a Gaussian $(\alpha>2)$ while the latter applies to "sub-Gaussian" entire functions with $\alpha<2$ and also gives new information about singular functions with $\alpha<1$. In the junction case $\alpha=2$, it is known from examples that it is possible for $a_{n} \sim O\left(e^{-n \log n}\right)$ if $f(z)$ decays as $e^{-z^{2} / 2}$ precisely but no better than $a_{n} \sim O\left(e^{-p n}\right)$ where $p$ is a constant when $f(z)$ decreases as $e^{-q z^{2}}$ with $q \neq 1 / 2$. When $\alpha \neq \rho$, some overlap between Theorems 1 and 2 is possible, and one should obviously use whichever provides the tighter bound. Theorem 3 illustrates the fact that $\rho>\alpha$, for a given $\alpha$, has an adverse effect on the convergence rate.

Two principal conclusions emerge from this. First, although it is still possible for the Hermite series of $f(z)$ to converge exponentially fast even if its asymptotic behavior does not precisely match the $\exp \left(-z^{2} / 2\right)$ decay of the Hermite functions themselves, so long as $f(z)$ does decrease exponentially at some rate or other as $|z| \rightarrow \infty$ on the real axis, it is clear that the poorer the match is-the more $\alpha$ differs from 2 either above or below-the poorer the convergence of the Hermite series will be. 
Second, the convergence theory of Hermite functions is more complicated than that for power series or Chebyshev polynomials because the asymptotic form of the series coefficients must depend on $\alpha$, the exponent of real axis decay, as well as upon either the order $\rho$ (entire functions) or nearest singularity (singular functions) which by themselves would be sufficient to determine asymptotic coefficients for these other types of expansions.

Work now in progress suggests that when $\alpha=\rho$, the lower bounds proved here are also (changing the sign of $\epsilon$ ) upper bounds as well. The proof of this, however, is at present incomplete and involves a line of reasoning completely different from that employed here. It will therefore be left for a future article.

Acknowledgments. I wish to thank Professor Einar Hille for helpful correspondence and express the hope that this, his eighty-fifth year, may be as fruitful as the past twenty have been ( 8 books and 44 papers). I also thank the referee for his detailed comments, particularly for suggesting [8]. This work was supported by NASA Grant NSG-6209.

Department of Atmospheric and Oceanic Science

University of Michigan

Ann Arbor, Michigan 48109

1. M. ABRAMOWITZ \& I. A. STEGUN, Eds., Handbook of Mathematical Functions, Dover, New York, 1965. MR 29 \#4914.

2. J. P. BOYD, "Hermite polynomial methods for obtaining analytical and numerical solutions to eigenvalue problems in unbounded and spherical geometry," J. Comput. Phys. (Submitted.)

3. E. HILLE, "Contributions to the theory of Hermitian series," Duke Math. J., v. 5, 1939, pp. 875-936. MR 1, pp. 141-142, MF 827.

4. E. HILLE, "Contributions to the theory of Hermitian series. II. The representation problem," Trans. Amer. Math. Soc., v. 47, 1940, pp. 80-94. MR 1, 142.

5. E. HILLE, "A class of differential operators of infinite order. I." Duke Math. J., v. 7, 1940, pp. 458-495. MR 2, 184.

6. E. HILLE, "Sur les fonctions analytiques définies par des séries d'Hermite," J. Math. Pures Appl., v. 40, 1961 , pp. 335-342.

7. E. C. TITCHMARSH, The Theory of Functions, Oxford Univ. Press, London, 1939.

8. R. ASKEY \& S. WAINGER, "Mean convergence of expansions in Laguerre and Hermite series," Amer. J. Math., v. 87, 1965, pp. 695-708. 